\title{
Integration digitaler Assistenzsysteme für die industrielle Montage
}

\author{
Thimo Keller, Christian Bayer, Joachim Metternich, Stephanie Schmidt, \\ Mehrach Saki und Oliver Sträter
}

\subsection{Vorstellung der mitwirkenden Projektpartner}

Die Interdisziplinarität der Thematik erfordert eine enge Zusammenarbeit unterschiedlicher wissenschaftlicher Fachgebiete. Das wissenschaftliche Konsortium, bestehend aus dem Fachgebiet für Arbeits- und Organisationspsychologie der Universität Kassel, dem Institut für Produktionsmanagement, Technologie und Werkzeugmaschinen der Technischen Universität Darmstadt und der Gesellschaft für Personal- und Organisationsentwicklung vereint das erforderliche Fachwissen in den Bereichen Mensch, Technik, Wertschöpfungsprozess und Organisation.

Neben der Kooperation zwischen Experten der wissenschaftlichen Disziplinen ist eine enge Zusammenarbeit von Wissenschaft und Industrie erforderlich, um technische und organisatorische Lösungen zur Aufwertung von Arbeit in der Produktion in die praktische Anwendung zu bringen. Aus diesem Grund wurde das Konsortium für das Forschungsprojekt entsprechend mit Vertretern der benötigten Disziplinen aufgestellt. Die Expertise für den Einsatz moderner Kommunikationstechnologie und die softwareseitige Gestaltung der digitalen Medien steuerte das, auf Digitalisierungsprojekte spezialisierte, Softwareunternehmen Bright Solutions bei. Die beteiligten Anwenderunternehmen, deren Produktionsumgebungen im Rahmen des Projekts betrachtet

T. Keller $(\bowtie) \cdot$ C. Bayer $\cdot$ J. Metternich

Technische Universität Darmstadt, Institut für Produktionsmanagement,

Technologie und Werkzeugmaschinen, Darmstadt, Deutschland

S. Schmidt $\cdot$ M. Saki $\cdot$ O. Sträter

Universität Kassel, Fachgebiet Arbeits- und Organisationspsychologie,

Kassel, Deutschland 
wurden, brachten das entscheidende Wissen $\mathrm{zu}$ den Produktionsprozessen in das Konsortium. Durch die Betrachtung der Fertigung eines KMU in der Elektronikbranche (mikrolab), der Produkte eines Werkzeugmaschinenherstellers (DATRON) sowie der Leuchtenendmontage eines Konzerns (TRILUX) wurden unterschiedliche Rahmenbedingungen und Zielstellungen berücksichtigt. Diese Vielseitigkeit der Anwenderunternehmen und deren Herausforderungen unterstützten die Interdisziplinarität des Vorhabens, um die Entwicklung eines übertragbaren Modells zur ganzheitlichen Aufwertung von manuellen und teilautomatisierten Arbeitssystemen in der Produktion durch digitale Kommunikationstechnologie zu ermöglichen.

\subsection{Zielstellung des Forschungsprojektes IntAKom}

Zur Realisierung der Vision von ,hochflexiblen, wandlungsfähigen Wertschöpfungssystemen“ wird der Digitalisierung (Industrie 4.0) eine entscheidende Rolle zugeschrieben [2]. Neben den neuen technischen Möglichkeiten zur Umgestaltung der Wertschöpfungsnetzwerke werden die Auswirkungen auf die Beschäftigten in den produzierenden Betrieben oft nur am Rande betrachtet. Jedoch werden die unter dem Begriff Industrie 4.0 beschriebenen Entwicklungen massive Konsequenzen auf die industrielle Arbeit, ihre Organisationsformen sowie die Kompetenzanforderungen der Belegschaften haben [1]. Die Arbeitsaufgabe der Beschäftigten wird durch die zunehmende Flexibilisierung und Digitalisierung unter anderem folgendermaßen geprägt [4]:

- häufige Produktwechsel,

- kurzzyklischer, flexiblerer Wechsel der Arbeitsaufgabe,

- hochflexibler Einsatz der Beschäftigten,

- Taktunabhängigkeit,

- Zunahme der Problemlösungs- und Überwachungsfunktion

Neben einer sinkenden physischen Beanspruchung werden eine steigende Beanspruchung der Sinnesorgane/Nerven sowie steigende emotionale und mentale Beanspruchungen erwartet [4]. Zur Entlastung der Beschäftigten wird der lern- und gesundheitsförderlichen Arbeitsgestaltung von Montagearbeitsplätzen eine hohe Bedeutung zugeschrieben.

Durch die zunehmende Konfrontation der Beschäftigten mit einer komplexen Arbeitsumgebung und wechselnden Aufgaben wird der Bedarf an aktuellen - für die Arbeitsaufgabe notwendigen - Informationen steigen. Die individuellen Voraussetzungen der Beschäftigten - wie die Erfahrung und das Wissen - bestimmen, wie stark die Komplexität einer Aufgabe wahrgenommen wird und welchen Schwankungen die Qualität der Produkte unterliegt [3]. 
Eine unzureichende Informationsversorgung führt $\mathrm{zu}$ einer Vielzahl von Konsequenzen, von einem übermäßig hohen Aufwand bei der Informationsbeschaffung bis hin zu Fehlhandlungen. Mithilfe digitaler Assistenzsysteme können die Beschäftigten zur richtigen Zeit mit der gerade benötigten Information versorgt werden. Durch den Einsatz digitaler Medien als Assistenzsysteme besteht die Möglichkeit, Arbeitsinhalte $\mathrm{zu}$ erweitern, anzureichern und innovative Arbeitsstrukturierungskonzepte wie Jobrotation zu fördern. Darüber hinaus können die Beschäftigten stärker an der Beseitigung von Fehlern und Störungen beteiligt werden und die dabei gewonnenen Erfahrungen strukturiert in die Verbesserung der Prozesse einfließen lassen.

Das Forschungsprojekt IntAKom verfolgte die Entwicklung eines übertragbaren Gestaltungskonzepts für industrielle Arbeitsumgebungen. Hierbei stand die Unterstützung der Beschäftigten zur Steigerung der Leistungsfähigkeit in Bezug auf die Arbeitsaufgabe im Mittelpunkt. Es wurde ein übertragbares Modell zur systematischen und ganzheitlichen Aufwertung von manuellen und teilautomatisierten Arbeitssystemen in der Produktion durch digitale Kommunikationstechnologie entwickelt, erprobt und dokumentiert.

\subsection{Präsentation der Projektergebnisse}

\subsubsection{TRILUX}

Die TRILUX-Gruppe ist mit mehr als 5.500 Mitarbeitenden ein weltweit tätiger Anbieter technischer Lichtlösungen und der zugehörigen Elektronik. Das umfangreiche Produktportfolio umfasst konventionelle und insbesondere LED-Leuchten-Baureihen in hoher Varianz. Die Endmontage der Produkte erfolgt in verschiedenen Montagebereichen von Hand und teilautomatisiert. TRILUX legt großen Wert auf eine flexible Werkerführung.

Während des Montageprozesses werden sie durch das Assistenzsystem OptiMa (Optimized Manufacturing) unterstützend begleitet und haben die Möglichkeit zur Produktinformationsbeschaffung, sofern diese benötigt wird (Montageprotokolle, Schaltpläne, Stücklisten). Die Entwicklung des Assistenzsystems war zu Projektbeginn bereits weit fortgeschritten. Der technische Prototyp wurde innerhalb des Projekts IntAKom analysiert, um Verbesserungspotenziale zu identifizieren. Diese wurden bei der Weiterentwicklung berücksichtigt. Weiterhin konnte das Forschungsprojekt mit der Organisation und Durchführung von Workshops zur Einführung des Assistenzsystems unterstützen.

Im Rahmen von IntAKom wurden zu Beginn strukturierte Interviews mit einzelnen Mitarbeitenden, ArbeitnehmervertreterInnen und Führungskräften sowie eine Befragung der Beschäftigten anhand eines umfangreichen Fragebogens in den dazugehörigen Abteilungen durchgeführt. Zudem wurden Eyetracking-Aufnahmen und eine Wertstromund Informationsbedarfsanalyse eingesetzt, um die Ausgangssituation an den Montagearbeitsplätzen zu bewerten. Die abgeleiteten Projektschwerpunkte bei TRILUX waren 
„Zusammenarbeit im Team“, „verlässliche Daten“, „situations- und produktbezogener Informationsbedarf“, „Ergonomie“, „MitarbeiterInnenintegration bei Veränderungsprozessen“ sowie ,aufwendige, papierbasierte Prozesse“. In einzelnen Workshops wurden diese Schwerpunkte systematisch bearbeitet und sowohl die IST-Zustände als auch die SOLL-Zustände definiert. Aus diesen Schwerpunkten wurden in einem weiteren Schritt Arbeitspakete definiert und mit Prioritäten versehen. Es entstanden entsprechende Arbeitspakete zur Adressierung der genannten Projektschwerpunkte, an denen im weiteren Projektverlauf gearbeitet wurde.

Mit Hilfe des entstandenen Assistenzsystems soll den Mitarbeitenden die Möglichkeit einer selbstständigen Qualifizierung gegeben werden. Zu diesem Zweck stand bei der Entwicklung der digitalen Oberflächen des Assistenzsystems das Lernen durch eine intuitive und einfache Bedienung im Fokus. Während der Entwicklung des Assistenzsystems wurden die Mitarbeitenden von Anfang an in den Entwicklungsprozess einbezogen. Aufgrund der großen Produktvielfalt war die Einführung eines Assistenzsystems notwendig, um die Qualität der Produkte zu sichern. Durch das Assistenzsystem können die Mitarbeitenden bei komplexen Arbeitsschritten gezielt mit den für sie notwendigen Inhalten unterstützt werden. Zudem wurde die Kommunikation mit anderen Abteilungen durch entsprechende Schnittstellen innerhalb des Assistenzsystems verbessert, um die Beschäftigten von der Vormontage und der Prüfung bis hin zum Verpacken des Produkts optimal zu unterstützen. Das Assistenzsystem ist Bestandteil der u-förmigen Montageinseln und umfasst Berührungsbildschirme, Tablets, kabellose Handscanner und eine moderne Prüftechnik. Das Assistenzsystem ist in Abb. $15.1 \mathrm{zu}$ sehen.

OptiMa ist direkt mit dem ERP-System verbunden und bezieht darüber die Arbeitsaufträge und entsprechende Daten. Die Mitarbeitenden können ihre Aufträge eigenständig am Arbeitsplatz auswählen. Bereits bei der Vorbereitung/beim Rüsten des

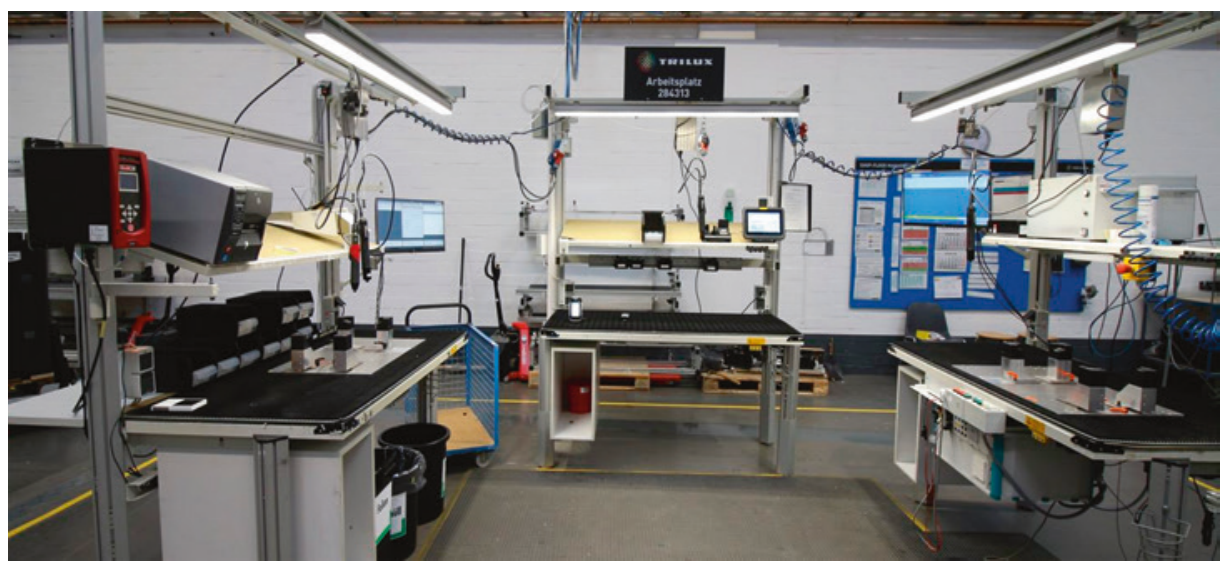

Abb. 15.1 Arbeitsplatz „OptiMa“ 
Arbeitsplatzes unterstützt das Assistenzsystem durch die Bereitstellung digitaler Stücklisten auf mobilen Handscannern. Die digitale Stückliste wird beim Buchen des Auftrags direkt im System zur Verfügung gestellt. Die Mitarbeitenden können die für die Auftragserfüllung benötigten Teile direkt vor Ort abscannen und so unnötige Suchprozesse durch Übersehen von Teilen vermeiden, da das System sowohl die bereits gescannten als auch die noch ausstehenden Teile anzeigt. Während der Montage kann das System die Mitarbeitenden unterstützen; es führt diese jedoch im Sinne eines klassischen Werkerführungssystems ausschließlich bei qualitätskritischen Prozessschritten. Die Mitarbeitenden können sich die Montageschritte vor Ort anzeigen lassen, werden jedoch vom Assistenzsystem nicht durch jeden einzelnen Schritt geführt. Dadurch soll vermieden werden, dass die Tätigkeiten Schritt für Schritt vorgegeben und somit kleinteiliger und monotoner werden. Alle benötigten Informationen, wie Montagepläne, Montagevideos usw. sind im System hinterlegt und können direkt am Arbeitsplatz abgerufen werden. Hierdurch wird eine schnellere Einarbeitung der Beschäftigten bei der Einführung neuer Produkte ermöglicht.

In einem letzten Schritt wurden wiederum eine Wertstrom- und Informationsbedarfsanalyse sowie eine MitarbeiterInnenbefragung durchgeführt, um Veränderungen durch die Einführung von OptiMa aufzeigen/bewerten zu können. Dabei wurden bei der Befragung der Beschäftigten vor allem die Themen „Entgelt“ und „Belastung der Mitarbeitenden“ bei der Einführung digitaler Assistenzsysteme angesprochen, was Verbesserungspotenziale für die Zukunft aufzeigt. Auf der anderen Seite konnte durch das Arbeiten mit OptiMA konkret die Gruppenbetreuer entlastet werden, da Nebentätigkeiten, wie z. B. Ausdrucke oder das Erklären von Leuchten minimiert wurden bzw. komplett wegfallen. Darüber hinaus wird eine Kostenersparnis durch die Programmierung der elektronischen Betriebsgeräte direkt an den Arbeitsplätzen erzielt. Auch die administrativen Kosten werden durch OptiMA erheblich gesenkt.

\subsection{2 mikrolab}

mikrolab ist ein Systemdienstleister für die Entwicklung und Produktion von kundenspezifischen elektronischen Geräten und Systemen in den Bereichen Navigation, Telemetrie und Betriebssystem. Das primäre Ziel im Rahmen des Forschungsvorhabens IntAKom ist es die zahlreichen unterschiedlichen Montageprozesse auf Basis von digitalen Hilfsmitteln zu optimieren und die betroffenen Mitarbeitenden bei den zunehmend komplexer werdenden Aufgaben adäquat zu unterstützen.

Im Zuge der Planung und Evaluationsphase für die Vorbereitung der späteren Einführung und Integration des digitalen Assistenzsystems in bestehende Programme und ERP-Systeme galt es für mikrolab u. a. die in nachfolgender Tab. 15.1 aufgeführten Fragen und darauf bezogenen Rahmenbedingungen zu berücksichtigen. 
Tab. 15.1 Zu beantwortende Fragen in der Planungsphase

\begin{tabular}{l|l}
\hline Fragen & Rahmenbedingungen \\
\hline $\begin{array}{l}\text { Sind die grundlegenden Arbeitsabläufe und } \\
\text { Prozesse ausreichend definiert? }\end{array}$ & $\begin{array}{l}\text { Ein Assistenzsystem wird schlechte und unvoll- } \\
\text { ständige Prozesse nicht kompensieren }\end{array}$ \\
\hline $\begin{array}{l}\text { An welchen Stellen agiert das Assistenz- } \\
\text { system autark und an welchen interagiert es mit } \\
\text { anderen Programmen? }\end{array}$ & $\begin{array}{l}\text { Die technische Machbarkeit auf Basis von } \\
\text { wirtschaftlichen Aspekten ist zu prüfen. Bei- } \\
\text { spielsweise ob zusätzlicher Programmierauf- } \\
\text { wand an bislang nicht berücksichtigter Stelle zu } \\
\text { erwarten ist }\end{array}$ \\
\hline $\begin{array}{l}\text { Von wo werden die Informationen abgerufen } \\
\text { und wo werden neue Informationen } \\
\text { gespeichert? }\end{array}$ & $\begin{array}{l}\text { Eine gemeinsame Datenbasis mit ent- } \\
\text { sprechenden Schnittstellen ist zu definieren }\end{array}$ \\
\hline $\begin{array}{l}\text { Ändern sich durch die Einführung des } \\
\text { Assistenzsystems bestehende Prozesse? }\end{array}$ & $\begin{array}{l}\text { Sich ändernde Kompetenzen, Arbeitsabläufe } \\
\text { oder z. B. Kommunikationsstrukturen sind zu } \\
\text { berücksichtigen }\end{array}$ \\
\hline $\begin{array}{l}\text { Welche Hilfsmittel sind in bestehenden oder } \\
\text { zukünftigen Tools bereits vorhanden, welche } \\
\text { Lücke soll das Assistenzsystem schließen? }\end{array}$ & $\begin{array}{l}\text { Redundanzen sind zu vermeiden. Oftmals } \\
\text { existieren bereits Hilfsmittel, doch sind diese } \\
\text { entweder nicht bekannt oder nicht genutzt }\end{array}$ \\
\hline $\begin{array}{l}\text { Werden für das Assistenzsystem spezielles } \\
\text { Equipment oder spezielle Lizenzen benötigt? }\end{array}$ & $\begin{array}{l}\text { Es ist zu prüfen, ob sich die Anschaffung unter } \\
\text { wirtschaftlichen Gesichtspunkten lohnt }\end{array}$ \\
\hline $\begin{array}{l}\text { Wo existiert Verbesserungspotenzial und } \\
\text { welche Vorteile werden mit der Einführung des } \\
\text { Assistenzsystem im Detail erwartet? }\end{array}$ & $\begin{array}{l}\text { Nur wenn bekannt ist, was verbessert werden } \\
\text { kann und soll, kann auch entsprechend ver- } \\
\text { glichen und nachgesteuert werden }\end{array}$ \\
\hline
\end{tabular}

Auf Basis dieser Fragen wurde innerhalb des Projekts IntAKom damit begonnen die bei mikrolab existierenden Montageprozesse und den dazugehörigen Informationsfluss an ausgewählten Beispielen zu analysieren und Prozesse mit Verbesserungspotenzial aufzudecken. Vor allem bei komplexen Prozessen wurde gezeigt, dass den Mitarbeitenden zwar oftmals viele Informationen zur Verfügung stehen, die für den aktuellen Arbeitsschritt passenden Daten jedoch mit einem gewissen Aufwand selektiert oder beschafft werden müssen. Die nachfolgende Bewertung der zu dem Zeitpunkt aktuellen Prozesslandschaft und eine Soll-Ist-Analyse, um die notwendige digitale Assistenz bedarfsgerecht $\mathrm{zu}$ konzipieren, führten $\mathrm{zu}$ der Entscheidung ein neues ERP Systems zur Schaffung einer konsistenten Datenbasis einzuführen. Das geplante Assistenzsystem sollte parallel dazu konzipiert und am Ende in das ERP-System integriert werden.

Um ein geeignetes System auszuwählen und dessen Einführung vorzubereiten wurden die innerhalb von IntAKom identifizierten und zu optimierenden Punkte in 5 Kategorien unterteilt und jeweils separat beleuchtet. Da die Einführung eines ERP Systems nicht innerhalb der Projektlaufzeit abgeschlossen werden konnte, galt es bei jedem Punkt zu bewerten, ob dessen Umsetzung bzw. Optimierung mittelfristig erfolgen kann, oder ob eine Interimslösung im aktuellen Verwaltungssystem von mikrolab notwendig ist. 


\section{Auftragssteuerung}

Als erster zentraler Schritt im Produktionsprozess wurde die projektübergreifende Auftragssteuerung betrachtet. Diese bildet die Basis für die weiteren Montageprozesse und ist daher als essenziell wichtig zu bewerten. Das bestehende Verwaltungssystem wurde mit einer visuellen Übersicht über alle anstehenden Aufträge erweitert. Die dafür notwendige Datenbasis findet sich in einer gemeinsamen Datenbank wieder und kann deshalb in zukünftige Systeme leicht übernommen werden. Bei der späteren Migration ist geplant folgende Verbesserungspotenziale und Planungsmechanismen zu berücksichtigen, welche das neue ERP-System per se bereits enthalten soll:

- Einlastung neuer Aufträge gegen verfügbare Kapazität,

- bessere Prüfung verfügbarer Materialien,

- bessere Bereitstellung auftragsrelevanter Dokumente durch ein DatenbankManagementsystem,

- bessere Steuerung der Aufgaben durch Arbeitsgangstruktur.

\section{Arbeitspläne}

Ein Arbeitsplan beinhaltet die relevanten projektspezifischen Informationen, welche Arbeitsschritte bei einem Produkt von welcher Abteilung, in welcher Reihenfolge, mit welchen Hilfsmitteln (Werkzeugen), in welcher Zeit und mit welchen Zusatzinformationen durchzuführen sind. Im Fall von mikrolab ist dies die Darstellung der jeweiligen Baugruppe mit dazugehörigen Arbeitsgängen, Funktionsbereichen (Belegungseinheiten) und weiterführenden Dokumenten in einem Filesystem auf dem Server.

Aufgrund des im Rahmen von IntAKom identifizierten, vergleichsweise hohen Verbesserungspotenzials wurde die Einführung der digitalen Arbeitspläne bereits im aktuellen ERP-System umgesetzt, wobei darauf geachtet wurde, gleichzeitig eine Basis für die spätere Datenmigration zu schaffen. Die Arbeitspläne können von den Mitarbeitenden jederzeit am jeweiligen Arbeitsplatz selbstständig eingesehen werden.

\section{Prozessbeschreibungen}

Eine wichtige Voraussetzung für die Einführung von Assistenzsystemen oder neuen Tools ist zu definieren, an welcher Stelle diese in bestehende Prozesse eingreifen. $\mathrm{Zu}$ diesem Zweck wurde bei mikrolab die für die Visualisierung der Prozessbeschreibungen nötige Basis geschaffen. Eine Prozesslandkarte auf Basis des Tools „viflow“ wurde auf dem Server veröffentlicht, ist im Intranet erreichbar und wächst sukzessiv. Der übergeordnete Prozess „Produktion“ ist in Abb. 15.2 dargestellt.

\section{Betriebsdatenerfassung}

Ein integriertes (Assistenz)System sollte neben der Bereitstellung von relevanten Informationen den Mitarbeitenden auch die Möglichkeit bieten, ihre Aufgaben möglichst effizient zu erhalten und rückmelden zu können. Um dies bei mikrolab zu erreichen 


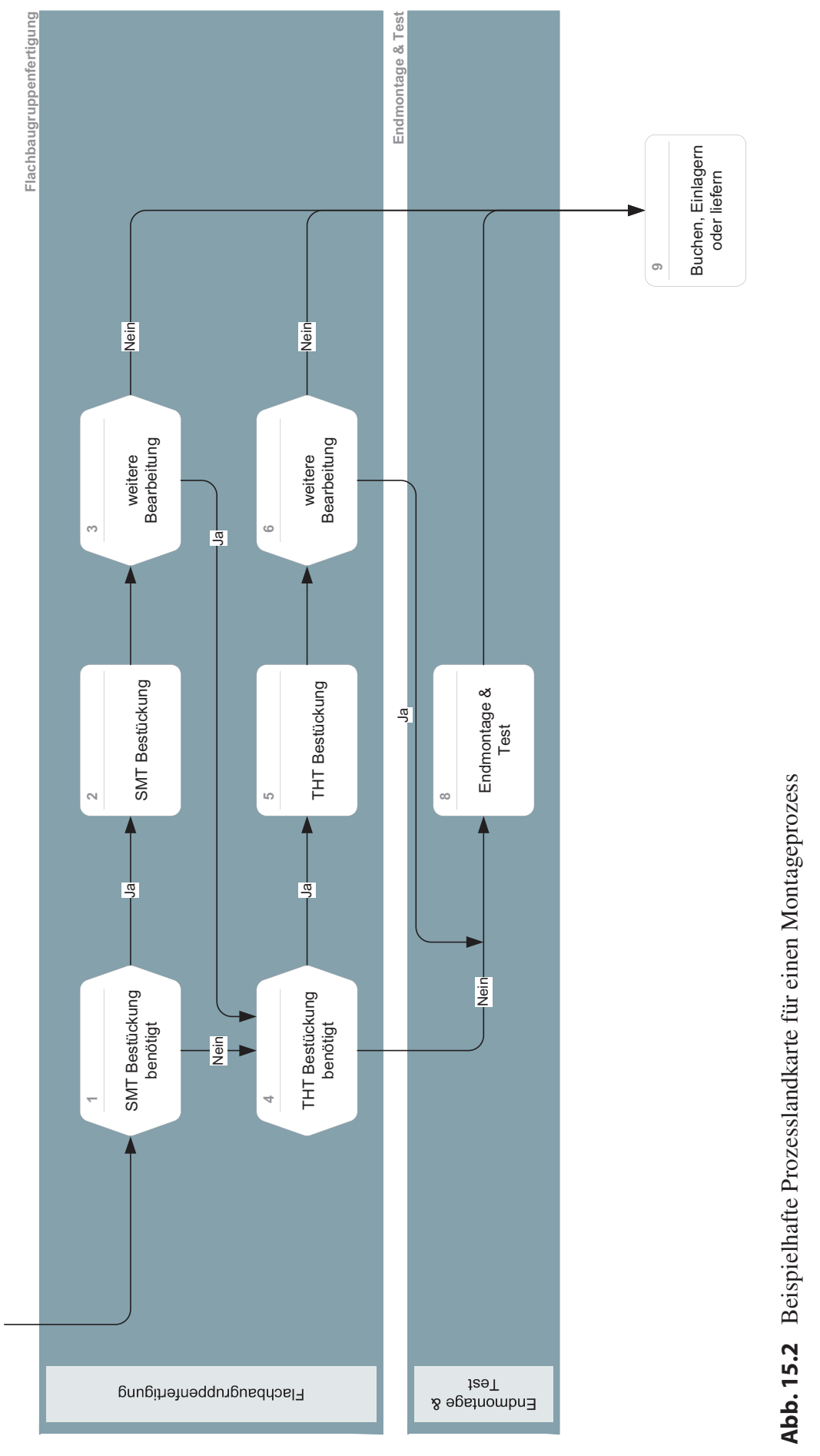


wird der Fokus u. a. auf die Flexibilität der BDE-Terminals gelegt. Diese sollen neben der erwähnten typischen Rückmeldung auch die relevanten Informationen an den Ort der Wertschöpfung transportieren und dort visualisieren. Insgesamt werden folgende Services integriert:

- Personalzeiterfassung (PZE)

- Employee Self Services (ESS)

- Betriebsdatenerfassung (BDE)

Zusammenfassend ist festzuhalten, dass sich für mikrolab die Schaffung einer konsistenten Prozess- und Datenstruktur als Grundlage einer erfolgreichen Einführung von neuen Assistenz- bzw. Hilfsprogrammen darstellte. Die ganzheitliche Betrachtung und Aufarbeitung der relevanten unternehmensspezifischen Abläufe ermöglicht eine spätere Integration von neuen Datenflüssen in bestehende Systeme und die Vernetzung mehrerer Tools.

Bereits die ersten Umsetzungen der innerhalb von IntAKom identifizierten Verbesserungspotenziale im bestehenden ERP-System führten bei mikrolab dazu, dass Mitarbeitende relevante Informationen nun schneller erhalten, Laufwege verkürzt werden, Wartezeiten aufgrund von Rückmeldungen Vorgesetzter entfallen und somit Zeiten und Fehler innerhalb des Produktentstehungsprozesses reduziert werden konnten. Mit den bislang gewonnenen Erkenntnissen wird ab 2020 die Einführung des neuen ERPSystems betrieben, welches ab 2021 unternehmensweit für alle Prozesse zur Verfügung stehen soll. Durch die Integration der innerhalb von IntAKom identifizierten notwendigen Assistenzfunktionen wird das neue System eine Mischung aus Assistenz- und konventionellem ERP-System darstellen.

\section{Intranet}

Parallel zu den Umsetzungen im bestehenden Verwaltungssystem sowie den Planungen der Informationen und Prozesse, die im neuen ERP-System mit abzudecken sind, wurde gemeinsam mit dem Projektpartner Bright Solutions ein firmenspezifisches Intranet erstellt.

Eine Sammlung der dafür notwendigen bzw. sinnvollen Inhalte wurde gemeinsam mit der Universität Kassel definiert. Das Intranet enthält eine Sammlung relevanter und nützlicher Links bzw. Information und bietet zudem Features, die den Arbeitsalltag erleichtern und nicht in weiteren Tools enthalten sind. So sind dies u. a. die Verwaltung, die Zeiterfassung, der QM-Bereich und ein mikrolab-Wiki. Ebenso wird der Mitarbeitende in die Lage versetzt im Intranet mit anderen Beschäftigten zu kommunizieren und projektspezifisch Aufgaben anzulegen bzw. diese zu kommentieren. Eine Demoseite des Intranets ist in Abb. $15.3 \mathrm{zu}$ sehen. 


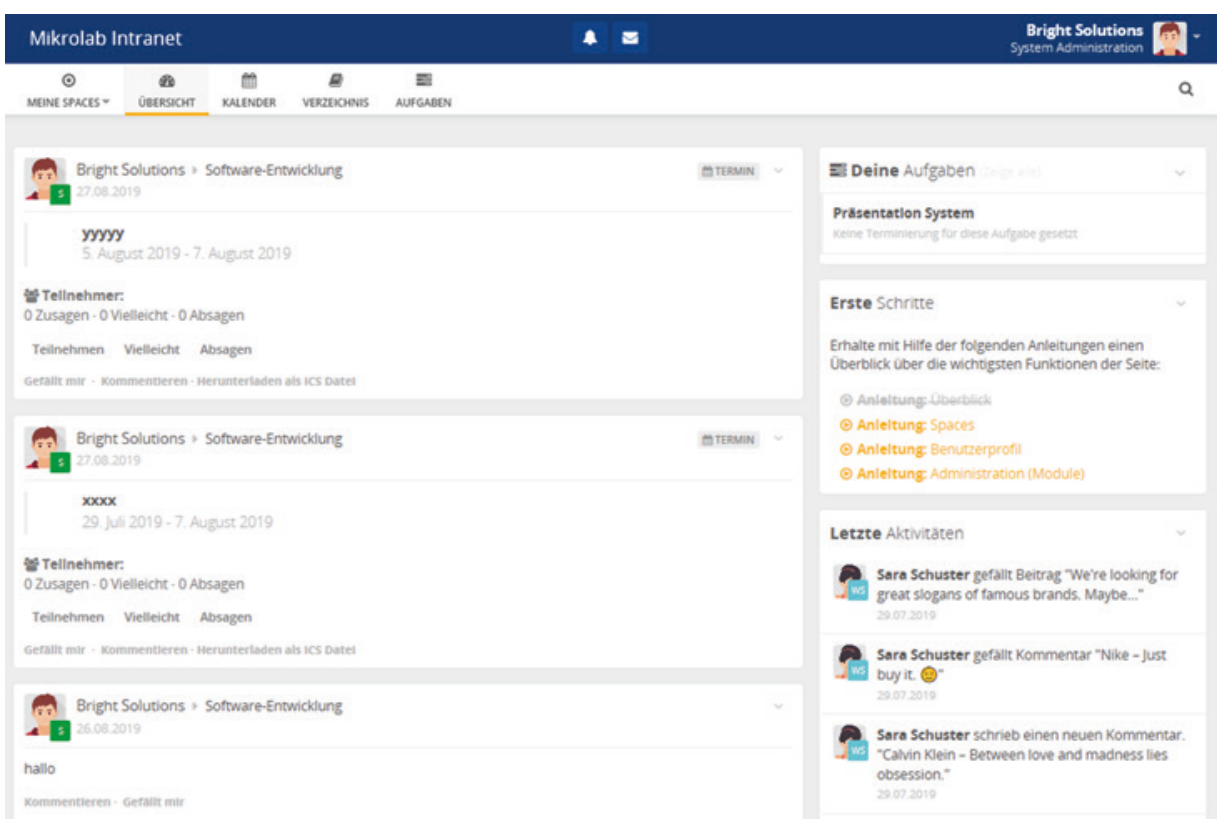

Abb. 15.3 Entwurf des realisierten Intranets

\subsubsection{DATRON}

Die DATRON AG ist ein international erfolgreicher Hersteller von CNC-Maschinen, Dental- CAD/CAM-Maschinen, Dosiersystemen und Zerspanungswerkzeugen. Für das mittelständische Maschinenbauunternehmen steht die Weiterentwicklung der eigenen Maschinen im Fokus (ca. $25 \%$ der Beschäftigten im Bereich Technologie). Der Schwerpunkt des Projektes bei DATRON lag auf der Bedienung und Instandhaltung der Maschinen im Betrieb, also auf der Optimierung der eigenen Produkte. Neben der Bedienung soll der Beschäftigte auch während des Montageprozesses der Werkzeugmaschine durch digitale Kommunikationstechnologie unterstützt und angelernt werden.

Die DATRON AG hat die Maschinensteuerung „NEXT“ in Eigenregie von Grund auf neu entwickelt und programmiert. Entwicklungsziel war eine optisch ansprechende und leicht verständliche Software mit einer kachelförmigen Anordnung der unterschiedlichen Funktionen auf einem großen Display. Basierend auf dem Plug and Play-Prinzip können so selbst Fräseinsteiger die 3-Achs CNC-Fräsmaschine auf Anhieb steuern. Die an ein Smartphone angelehnte Bedienung per Wischgesten macht das Fräsen intuitiv 
und verkürzt so die Phase des Einlernens. Im Rahmen des Projektes IntAKom wurde über Blickwinkelanalysen eine Ergonomie-Bewertung der Maschinensteuerung durchgeführt. In anschließenden Workshops wurden Verbesserungspotenziale, wie beispielsweise eine Feedbackfunktion für den Nutzer als vielversprechende Optimierung der Maschinensteuerung identifiziert und im Folgenden vom Entwicklerteam umgesetzt. Die Funktion bietet die Möglichkeiten, Bugs zu melden, Ideen, sowie Lob und Kritik mitzuteilen. Die Nachricht wird mit einem Screenshot des Bildschirms übermittelt, damit die Beschäftigten der Softwareentwicklung erkennen, an welchen Stellen im Bildschirm entsprechende Marker durch den Anwender gesetzt wurden. Unternehmensintern erhält die neu implementierte Funktion eine sehr gute Resonanz, da das Tool die Effizienz in der Zusammenarbeit zwischen Service, Technologen, Testing und Softwareentwicklung steigert.

Das zweite wesentliche Projektziel war die Verbesserung der produkt- und situationsbezogenen Informationsversorgung der Mitarbeitenden in der Endmontage. Nach intensiver Analyse der Montageprozesse mit den dazugehörigen Informationsflüssen wurden, in Zusammenarbeit zwischen den Forschungsinstituten und DATRON, unterschiedliche Verbesserungsansätze abgeleitet. Als wichtige Grundlage für die Informationsversorgung der Beschäftigten in der Montage wurde die Notwendigkeit erkannt die einzelnen Prozesse, Verantwortlichkeiten und Rollen klar zu definieren und abzugrenzen. In mehreren Workshops mit den Beteiligten wurde ein „Nordstern“ (SollZustand) für den gesamten Prozess der Auftragsabwicklung erarbeitet, an dem sich sämtliche Gestaltungsmaßnahmen und Änderungen orientieren.

Auf Basis einer umfangreichen Wertstrom- und Informationsbedarfsanalyse an den Montagestationen wurde der Ist- sowie der Idealzustand der Informationsversorgung der Beschäftigten erfasst. Das konkrete Ergebnis dieser Vorgehensweise waren Mockups, anhand derer der Aufbau und Inhalt einer digitalen Assistenz dargestellt wird. Diese Mockups zeigten pro Arbeitsschritt die notwendigen Informationen für die Beschäftigten auf und dienten als Grundlage für die Entwicklung der Software durch den Projektpartner Bright Solutions.

Eine Herausforderung lag in der Auswahl der Hardware für die Arbeitsumgebung der MontagemitarbeiterInnen. Die vorbereitenden Gespräche mit den Beschäftigten der Montage führten zu der Entscheidung, einen Werkstattwagen so auszustatten, dass sich darauf alle technischen Mittel für eine optimale Nutzung des Assistenzsystems platzieren lassen. Die Konstruktionszeichnung für die benötigte Vorrichtung wurde kurzerhand selbst erstellt und intern gefertigt. Der so erweiterte Werkstattwagen ist in Abb. $15.4 \mathrm{zu}$ sehen. 


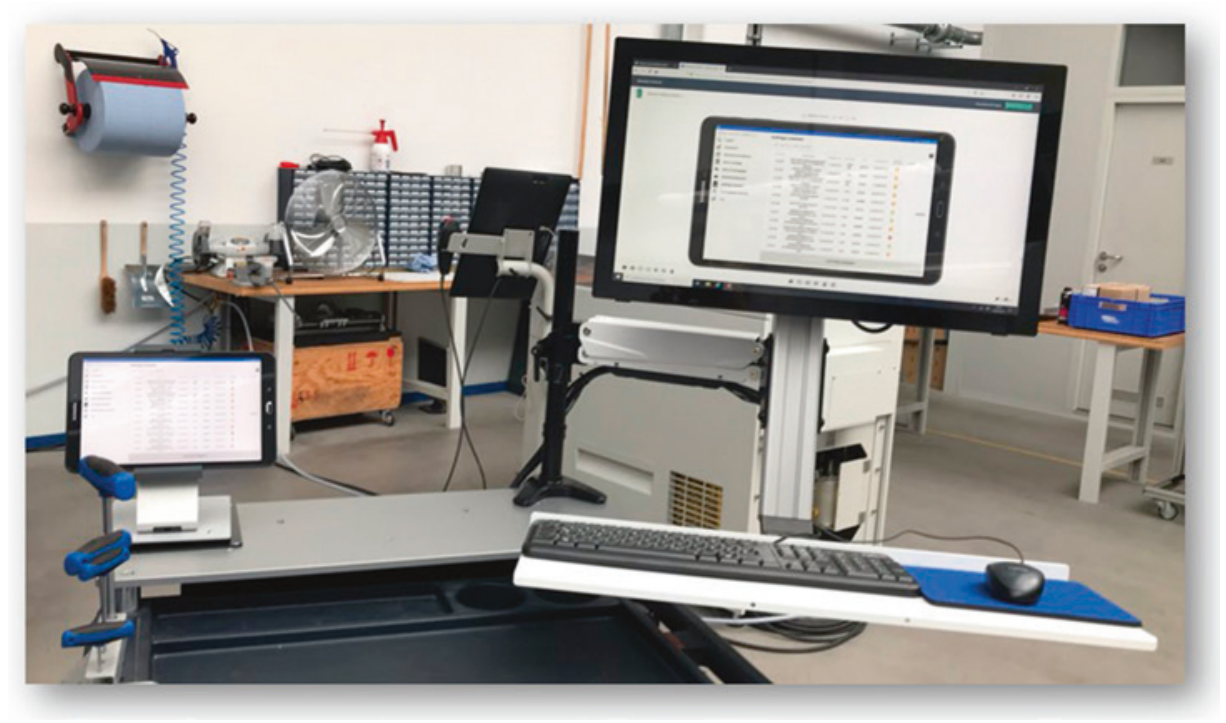

Abb. 15.4 Digitale Assistenz auf dem Montagewagen bei DATRON

Das Tablet ist an einem Magnetfuß befestigt und kann dadurch flexibel während der Montage mitgenommen und an der Maschinenkabine befestigt werden. Dies ist in Abb. $15.5 \mathrm{zu}$ sehen.

Die so entstandene digitale Assistenz, bestehend aus selbst entwickelter Soft- und Hardware, wurde an einem Pilotarbeitsplatz implementiert und über mehrere Wochen genutzt. Durch Aufnahme der Arbeitszeiten mithilfe einer Betriebsdatenerfassung wurde ermittelt, dass nach Inbetriebnahme des Assistenzsystems der Produktivzeitanteil von $85 \%$ auf $94 \%$ angestiegen und zudem die absolute Anzahl von Störungen pro Auftrag von 46 auf 20 gesunken ist. Zudem sind die Beschäftigten begeistert vom Ergebnis des entstandenen Pilotarbeitsplatzes. Auf dieser Basis fiel die Entscheidung, das Assistenzsystem im Laufe des Jahres 2020 produktionsweit auszurollen und ab 2021 neue Funktionen wie beispielsweise 3D-Darstellungen bereit zu stellen.

\subsection{Zusammenfassung und Ausblick auf weitere Forschungsbedarfe}

Innerhalb des Forschungsprojekts IntAKom konnte durch die Erfahrungen aus den Analysen in den Praxisunternehmen mikrolab, DATRON und TRILUX und den stetigen Austausch der wissenschaftlichen Partner ein übertragbares Analysetool entwickelt werden. Durch dieses Tool wird die Ausgangssituation in einem beliebigen Produktionsunternehmen mit dem Fokus auf die Einführung digitaler Assistenz umfassend 


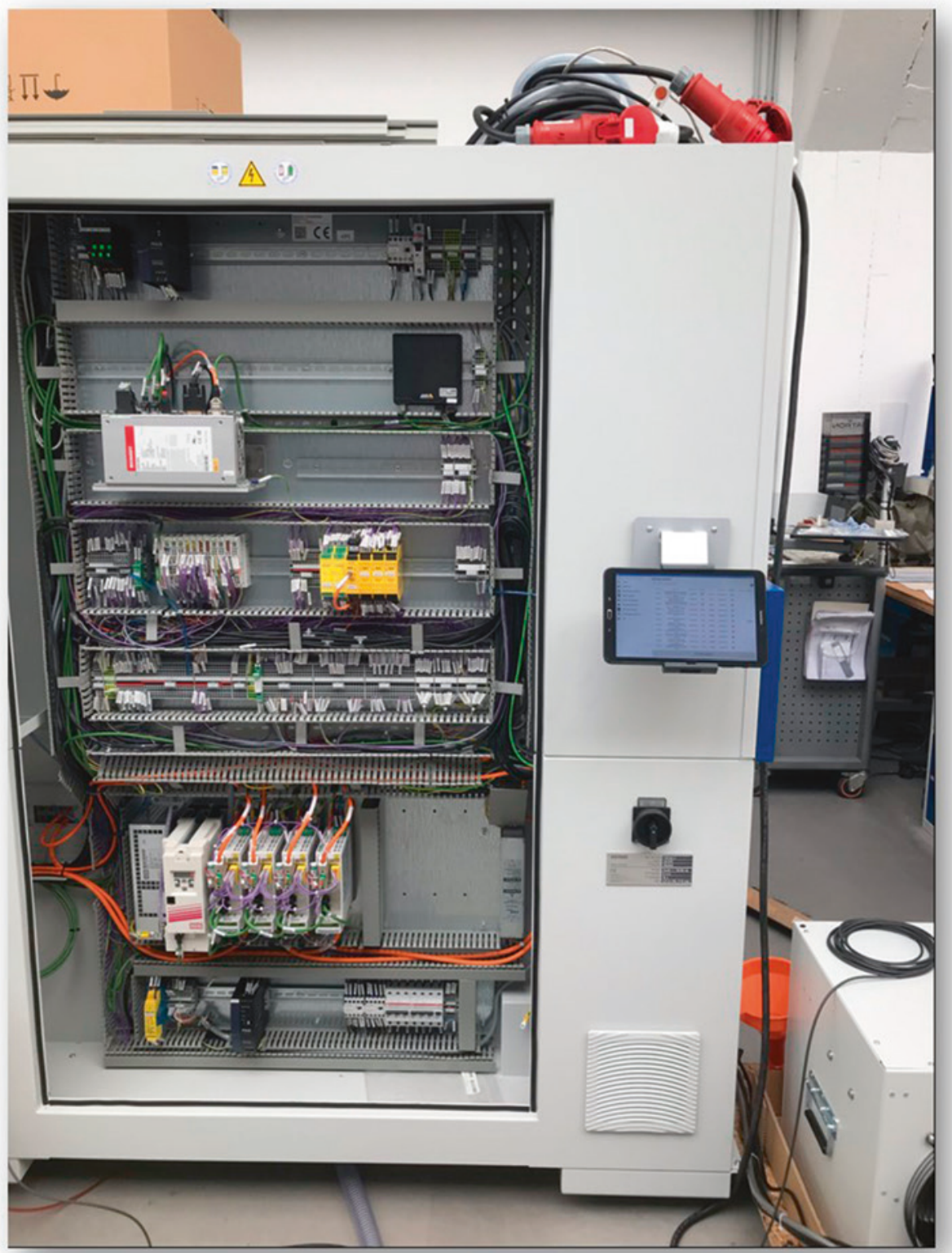

Abb. 15.5 Digitale Assistenz an der zu montierenden Werkzeugmaschine bei DATRON

beleuchtet. Im Speziellen werden die Bereiche Mensch, Technik, Wertschöpfungsprozess und Organisation analysiert, um die Situation ganzheitlich zu betrachten. Das Ergebnis stellt die Basis für die Entwicklung eines individuellen Assistenzsystems - angepasst an 
die Bedürfnisse des betrachteten Unternehmens - dar. Aufgrund der Allgemeingültigkeit der Vorgehensweise eignet sich diese für unterschiedliche Branchen, Prozesse und Arbeitsumgebungen.

Während der Einführung der verschiedenen digitalen Assistenzsysteme bei den drei Partnerunternehmen war besonders auffällig, dass unternehmensübergreifend die notwendige Voraussetzung für eine effektive Unterstützung der Beschäftigten eine strukturierte Datenbasis ohne Redundanzen ist. Neben der Konsolidierung der verwendeten IT-Systeme (MES, ERP, etc.) als Datenbasis für die digitale Assistenz, ist es zudem notwendig Prozesse, Verantwortlichkeiten und Wissensträger klar zu definieren und abzugrenzen. Nur wenn die unterschiedlichen Bereiche Mensch, Technik, Wertschöpfungsprozess und Organisation bestmöglich miteinander kooperieren, kann die gewünschte Unterstützung für die Beschäftigten erreicht werden.

Beim Ableiten der Anforderungen an digitale Assistenz wurden ebenfalls unternehmensübergreifend Ähnlichkeiten festgestellt. Im Fokus des Anforderungskatalogs der Anwenderunternehmen standen vor allem die Nutzerfreundlichkeit nach dem Leitfaden der DIN EN ISO 9241, die Verlässlichkeit und die Individualisierbarkeit des Systems. Zudem wurde bei der Umsetzung der Assistenz darauf geachtet die Beschäftigten während der Nutzung des Systems nicht zu eng zu führen, um die Handlungsspielräume zu erhalten oder sogar zu erweitern.

Aufgrund der sehr unterschiedlichen Ausgangssituationen in den drei Anwenderunternehmen innerhalb des Konsortiums konnte kein Ansatz für eine einheitliche Software-Lösung gefunden werden, bei der die digitale Assistenz mit geringem Aufwand für unterschiedliche Anwendungsfälle implementiert werden kann. Hier muss in Zukunft weitere Forschungsarbeit geleistet werden, um einen einheitlichen Ansatz in Form einer Software-Lösung zu entwickeln, der mit vertretbarem Aufwand verschiedene Anwendungsfälle aus unterschiedlichen Branchen adressiert. Wesentliche Herausforderungen werden hierbei die Schnittstellen zu der bereits bestehenden und teilweise diversen IT-Infrastruktur in produzierenden Unternehmen sein.

\subsection{Ein Leitfaden für die Praxis als nachhaltiges Projektergebnis}

Die gewonnenen Erkenntnisse zur ganzheitlichen Aufwertung von Arbeitssystemen wurden in dem Handbuch „Digitale Assistenz für die Produktion - Ein Leitfaden für die Bedarfsermittlung, Gestaltung und Einführung“ festgehalten. Übertragbare und erprobte Methoden bilden neben den umgesetzten Lösungen die Basis für den Leitfaden. Hierdurch verfügen Unternehmen künftig über hilfreiche Methoden sowie praxisnahe GoodPractice-Beispiele für eine gute und lernförderliche Gestaltung von Arbeitsorten und -prozessen. Das Handbuch wurde im Juni 2020 mit dem VDMA-Verlag veröffentlicht (ISBN: 978-3-8163-0737-2). 


\section{Projektpartner und Aufgaben}

- Technische Universität Darmstadt - Institut für Produktionsmanagement, Technologie und Werkzeugmaschinen (PTW)

Technologische Gestaltung von Arbeitssystemen für gute digitale Assistenz

- Universität Kassel - Institut für Arbeitswissenschaft und Prozessmanagement

Arbeits- und Tätigkeitsgestaltung 4.0 für gute digitale Assistenz

- ffw GmbH - Gesellschaft für Personal- und Organisationsentwicklung

Gestaltung von Arbeits- und Organisationskulturen für gute digitale Assistenz

- TRILUX GmbH \& Co. KG

Gestaltung von guter digitaler Assistenz für variantenreiche Serienprozesse

- mikrolab Entwicklungsgesellschaft für Elektroniksysteme GmbH

Gestaltung guter digitaler Assistenz für komplexe Fertigungsaufträge im Dienstleistungssektor

- DATRON AG

Ansätze zur Unterstützung der Handhabung und des Lernprozesses bei Montage, Inbetriebnahme, Vertrieb und Service von Werkzeugmaschinen

- Bright Solutions GmbH

Systematische Gestaltung digitaler Kommunikation durch moderne Web- und Mobile-Technologien

\section{Literatur}

1. acatech (Hrsg) (2016) Kompetenzentwicklungsstudie Industrie 4.0 - Erste Ergebnisse und Schlussfolgerungen, S. 9. https://www.acatech.de/publikation/kompetenzentwicklungsstudieindustrie-4-0-erste-ergebnisse-und-schlussfolgerungen/. Zugegriffen: 10. Juli 2019

2. Bauernhansl T (2014) Die Vierte industrielle Revolution. Der Weg in ein wertschaffendes Produktionsparadigma. In: Bauernhansl T, ten Hompel M, Vogel-Heuser B (Hrsg) Industrie 4.0 in Produktion, Automatisierung und Logistik. Springer Vieweg, Wiesbaden, S. 7

3. Blockus M-O (2010) Komplexität in Dienstleistungsunternehmen: Komplexitätsformen, Kosten- und Nutzenwirkungen, empirische Befunde und Managementimplikationen, 1. Aufl., Gabler Research, 28, Wiesbaden, 2010, S. 22

4. Dombrowski U, Riechel C, Evers M (2014) Industrie 4.0 Die Rolle des Menschen in der vierten Industriellen Revolution, in: Kersten, Wolfgang u. a. (Hrsg.), Industrie 4.0: Wie intelligente Vernetzung und kognitive Systeme unsere Arbeit verändern, Schriftenreihe der Hochschulgruppe für Arbeits- und Betriebsorganisation e.V. (HAB), Berlin, S 147 
Open Access Dieses Kapitel wird unter der Creative Commons Namensnennung 4.0 International Lizenz (http://creativecommons.org/licenses/by/4.0/deed.de) veröffentlicht, welche die Nutzung, Vervielfältigung, Bearbeitung, Verbreitung und Wiedergabe in jeglichem Medium und Format erlaubt, sofern Sie den/die ursprünglichen Autor(en) und die Quelle ordnungsgemäß nennen, einen Link zur Creative Commons Lizenz beifügen und angeben, ob Änderungen vorgenommen wurden.

Die in diesem Kapitel enthaltenen Bilder und sonstiges Drittmaterial unterliegen ebenfalls der genannten Creative Commons Lizenz, sofern sich aus der Abbildungslegende nichts anderes ergibt. Sofern das betreffende Material nicht unter der genannten Creative Commons Lizenz steht und die betreffende Handlung nicht nach gesetzlichen Vorschriften erlaubt ist, ist für die oben aufgeführten Weiterverwendungen des Materials die Einwilligung des jeweiligen Rechteinhabers einzuholen.

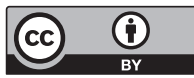

